\title{
Review
}

\section{Almanac 2013: stable coronary artery disease}

\author{
Shahed Islam, Adam Timmis* \\ NIHR Biomedical Research Unit, Barts and the London School of Medicine and Dentistry, London Chest Hospital, London, UK
}

Seminars in Cardiovascular Medicine 2013; 19:72-80

\section{Coronary heart disease in decline}

Epidemiological data from Europe, the USA and elsewhere in the developed world show a steep decline in coronary heart disease (CHD) mortality during the last 40 years [1]. Concern about levelling of mortality rates in younger adults [2] has been somewhat alleviated by data from The Netherlands showing that in men aged $<55$ years, rates of decline have again accelerated, increasing from only $16 \%$ in $1993-1999$ to $46 \%$ in 1999-2007 [3]. A similar pattern was observed in young women with rates of decline of $5 \%$ and $38 \%$ during the same time periods. This is encouraging, particularly in the context of data from Denmark and the UK showing declining mortality and also a sharp fall in standardised incidence rates for acute myocardial infarction indicating that coronary prevention, as well as acute treatments, has contributed to recent mortality trends $[4,5]$. Meanwhile an Australian study reminds us that myocardial infarction is but one of several manifestations of cardiovascular disease by reporting that decreasing incidence and recurrence rates for hospitalised CHD from 2000 to 2007 have also been seen for cerebrovascular and peripheral arterial disease [6].

However, the epidemiological news is not all good, and data from the UK show that the pernicious relationship between socioeconomic status (SES) and CHD has shown no tendency to go

\footnotetext{
* Corresponding address: Professor Adam Timmis, NIHR Biomedical Research Unit, Barts and the London School of Medicine and Dentistry, London Chest Hospital, London E2 9JX, UK.

E-mail: adamtimmis@mac.com (A. Timmis).

Acknowledgement of permission for re-publication: the article was first published in Heart (Islam S, Timmis A. Almanac 2013: stable coronary artery disease. Heart 2013 Nov;99(22):1652-7. doi: 10.1136/heartjnl-2013-304593. Epub 2013 Sep 5) and is republished with permission. Received 4 July 2013, accepted 13 August 2013, published online first 5 Sep 2013.
}

away in recent years, the gradients between top and bottom SES quintile groups for hospital admissions remaining essentially unchanged across the age range [7]. Whether this has contributed to the almost 3-fold risk of myocardial infarction associated with stillbirth and 9-fold risk associated with recurrent miscarriage in a recent German study is unclear because the investigators made no adjustment for SES [8]. Nor is it clear if SES has contributed to the persistent ethnic differences in both US and UK studies of CHD mortality although other factors appear also to be important. Thus, African-American men have greater exposure to CHD risk factors than Caucasians and, when adjustment is made for this, their susceptibility to CHD is no greater, although mortality rates are twice as high [9]. For African-American women, incidence and mortality rates are higher than their Caucasian counterparts. These findings suggesting that exposure to risk factors contributes to ethnic differences in the incidence of CHD are to some extent reflected in a recent report from the Health Survey for England in which 13,293 Caucasian and 2120 S Asians consented to mortality follow-up [10]. Physical inactivity was more frequent in S Asians compared with Caucasians $(47 \%$ vs $28 \%)$ and explained $>20 \%$ of their excess CHD mortality. Certainly, the emerging consensus is that the excess CHD mortality among UK S Asians is driven almost entirely by their increased susceptibility to disease and not by increased case-fatality rates [11].

\section{Diagnosis of stable coronary artery disease}

The recent AHA/ACC guideline update [12] emphasised the importance of individualising the diagnostic workup based on the estimated probability of coronary artery disease. In this respect, it mirrored an earlier National Institute of Clinical Excellence (NICE) guideline on chest pain 
diagnosis [13], but there were important differences in the recommendations for non-invasive testing, the new AHA/ACC guideline preferring the exercise ECG as the initial diagnostic approach for most patients, (NICE had previously counselled against use of the exercise ECG based on its relatively poor diagnostic performance) with pharmacologic radionuclide, cardiac MRI or stress echocardiography testing in reserve for patients unable to exercise. Recommendations for cardiac CT coronary angiography (CTCA) were cautious, and invasive angiography was recommended for diagnostic purposes only if the results of non-invasive testing suggested a high likelihood of severe 3-vessel or left main coronary artery disease, and the patient was willing to undergo revascularisation. In general, therefore, the AHA/ACC guideline update was less prescriptive than the earlier NICE guideline, perhaps partly because it put less emphasis on the cost effectiveness of its recommendations.

\section{Management of stable coronary artery disease}

The recent NICE guideline [14] recommended initial treatment with a short-acting nitrate and a $\beta$-blocker and/or a calcium channel blocker for control of angina plus aspirin and a statin for secondary prevention. Lifestyle measures were also emphasised. For patients with continuing symptoms cardiac catheterisation with a view to revascularization was recommended, additional antianginal treatment (long-acting nitrates or one of the newer agents) only being indicated for patients unsuitable for revascularisation. It was further recommended that the mode of revascularisation (percutaneous coronary intervention (PCI) versus coronary artery bypass grafting (CABG)) should best be determined by a multidisciplinary group, a recommendation that has also been emphasised by European guideline groups [15], bearing in mind the potential for prognostic benefit from CABG in patients with complex multivessel and left main stem disease, particularly those with diabetes [16]. For patients with symptoms adequately controlled with medical treatment, the guideline recommended discussion of the potential for prognostic improvement with CABG. Those patients prepared to proceed to CABG might then be offered diagnostic cardiac catheterisation to rule out complex multivessel and left main stem disease, which a recent metaanalysis reported in as many as 36\% (18.5-48.8\%) of cases of stable coronary disease selected for cardiac catheterization [17].

\section{Secondary prevention of stable coronary disease}

The scope for improving secondary prevention in patients with stable coronary artery disease has been emphasised in two recent reports. In The multinational REduction of Atherothrombosis for Continued Health (REACH) Registry, 20,588 symptomatic patients were analysed for 'good control' of cardiovascular risk factors, defined as three to five of systolic blood pressure < $140 \mathrm{mmHg}$, diastolic blood pressure $<90 \mathrm{mmHg}$, fasting glycaemia $<110 \mathrm{mg} / \mathrm{dl}$, total cholesterol $<200 \mathrm{mg} / \mathrm{dl}$, non-smoking [18]. Only 59.4\% had good control of risk factors at baseline, but this was associated with lower mortality (OR 0.89; 95\% CI 0.79-0.99) at 36 months, compared with poor control. In the UK ASPIRE-2-PREVENT survey, 676 patients with CHD (25.6\% women) had the following rates of major risk factors: smoking $14.1 \%$, obesity $38 \%$, physical inactivity $83.3 \%$, blood pressure $\geq 130 / 80 \mathrm{mmHg}$, total cholesterol $\geq 4 \mathrm{mmol} / \mathrm{l}$ and diabetes $17.8 \%$, leading the authors to conclude that there is considerable potential for reducing cardiovascular risk in these patients and thereby improve prognosis [19].

\section{Clopidogrel}

The availability of low-cost generic clopidogrel prompted a NICE review of its cost effectiveness which recommended it should now supersede aspirin in certain high-risk groups, namely patients with multivascular disease, peripheral vascular disease and myocardial infarction [20]. However, clopidogrel is metabolised by enzymes in the hepatic cytochrome P450 (CYP) system, and variability in its antiplatelet activity may occur because the activity of these enzymes is influenced by common genetic variations, and also by a number of commonly used drugs. Several studies have reported loss-of-function alleles in CYP2C19 that result in reduced activation of clopidogrel [21] and a modest lowering of antiplatelet activity [22] which have been associated with an increased risk of cardiovascular events in some meta-analyses [23]. Conversely, gain-of-function alleles have been associated with reduced cardiovascular risk among clopidogrel-treated patients [24]. A recent meta-analysis, however, has commented on the tendency of small studies to bias conclusions about the way genetic variants influence clinical outcomes, and in larger studies of clopidogrel therapy with $\geq 200$ outcome events found no effect of loss-of-function alleles on cardiovascular risk [25]. At present, therefore, there seems to be no compelling indication for genetic testing to guide clopidogrel treatment although the topic remains a subject of ongoing 
debate. Also debated is the interaction of clopidogrel with some commonly used drugs, particularly proton pump inhibitors (PPI) and amlodipine. A recent meta-analysis of studies of PPIs in patients treated with clopidogrel found clear evidence of reduced platelet activity but although clinical outcomes appeared adversely affected by the interaction, the authors urged cautious interpretation, pointing out the heterogeneity caused by retrospective studies. When analysis was restricted to prospective studies of PPIs and clopidogrel, adverse clinical consequences could no longer be demonstrated (OR $1.13(0.98-1.30)$ ) [26]. Similarly, the clinical impact of amlodipine on responsiveness to clopidogrel remains uncertain. Certainly, there is evidence of interaction, and in one study of 1258 patients receiving clopidogrel, amlodipine administration was associated with higher on-treatment platelet reactivity only in those patients with a loss-of-function P450 (CYP) genotype ( $249 \pm 83$ vs $228 \pm 84$ P2Y12 reaction units), and this was associated with a higher incidence of cardiovascular events ( $4.6 \%$ vs $0.6 \%$ ) [27]. However, in a more recent randomised trial, platelet function in 98 patients with stable coronary artery disease taking clopidogrel was similar regardless of amlodipine therapy [28]. At present, therefore, there is no guideline recommendation about concomitant prescription of these drugs in patients taking clopidogrel.

\section{Statins, niacin and cholesteryl ester transfer protein (CETP) inhibitors}

The benefits of statins for secondary prevention in patients with stable coronary artery disease are well established. Cardiovascular endpoints are reduced in proportion to the degree of LDL-cholesterol reduction, probably in response to stabilisation and regression of atheromatous plaque. The capacity for plaque regression has recently been confirmed by serial IVUS examination in 1039 patients with stable coronary disease randomised to rosuvastatin $40 \mathrm{mg}$ daily or atorvastatin $80 \mathrm{mg}$ daily [29]. Atheroma volume during the 2-year monitoring period decreased by an average of about $1 \%$ in both groups, more than previously reported with less intensive statin regimens. However, additional clinical benefits of niacin have now been unequivocally ruled out in the AIM-HIGH trial in which 3414 patients with stable cardiovascular disease taking statins were randomised to receive niacin $(n=1718)$ or placebo $(n=1696)$ [30]. Although niacin significantly increased HDL cholesterol and lowered triglycerides, differences in the primary endpoints (a composite of adverse coronary events, strokes and revascularisation) were negligible, occurring in $16 \%$ of patients in each group. The trial was stopped after an average follow-up of 3 years when it became clear HDL raising therapy with niacin was clinically ineffective. All hopes for HDL raising therapy are now invested in CETP inhibitors, and despite safety concerns following the ILLUMINATE trial of torcetrapib [31], in which treatment was associated with increased mortality despite substantial HDL elevations, other CETP inhibitors are now entering phase III trials. A recent randomised trial of dalcetrapib in patients with acute coronary syndromes was disappointing with no reduction in the risk of recurrent coronary events despite a $>30 \%$ increase in HDL levels in the treatment group [32]. An efficacy and safety trial of anacetrapib in patients with, or at high risk of, stable coronary disease was favourable, although not powered for clinical outcomes [33], and evacetrapib has now entered the arena with a recent study showing effective HDL raising without the adverse effects on blood pressure seen with torcetrapib and, to a lesser extent, dalcetrapib [34]. Whether any of these CETP inhibitors will improve clinical outcomes, however, remains unknown.

\section{Novel lipid-lowering drugs in clinical translation}

Conventional lipid-lowering therapies, even when combined with LDL-apheresis, are often insufficient to treat to guideline targets patients with familial hypercholesterolaemia (FH), an autosomal dominant disorder of lipid metabolism associated with accelerated coronary disease [35]. There is, therefore, considerable interest in novel therapies currently under investigation, particularly lomitapide, an oral inhibitor of microsomal transfer protein and monoclonal antibodies against PCSK9. A phase II study of lomitapide in homozygous $\mathrm{FH}$ showed a $50 \%$ reduction in LDL-cholesterol and, although gastrointestinal side effects were common, a useful role for the drug seems likely in these homozygous patients [36]. PCSK9 inhibitors have also produced $50-60 \%$ reductions in LDL-cholesterol values in clinical studies when added to statins and ezetimibe, but unlike lomitapide, are probably mainly effective in heterozygotic $\mathrm{FH}$ because they act through interference with LDL receptors which are dysfunctional or completely absent in homozygotes $[37,38]$. The expectation is that application of these new drugs will allow most patients with FH to achieve target concentrations of LDL cholesterol. An important component of FH management involves identification of other affected family members, and cascade screening using genetic testing has been reported as cost effective [39]. However, recent evidence suggests 
that polygenic disorders account for an appreciable proportion of FH cases [40], and this will limit the effectiveness of cascade screening to relatives of mutation-positive (monogenic) cases. In other patients, with cholesterol levels consistent with an FH genotype, more conventional primary care strategies [41] should remain the screening tool of choice, at least for the time being.

\section{Revascularisation in stable CAD}

\section{Percutaneous coronary intervention}

The COURAGE trial was a game-changer, showing that coronary stenting in patients with stable angina did not improve cardiovascular outcomes compared with optimal medical therapy (OMT) while quality-of-life benefits were short-lived [42, 43]. Now available is a meta-analysis comparing contemporary medical therapy and PCI in eight randomised trials involving 7229 patients with stable CAD [44]. Again, cardiovascular outcomes between the groups were similar during follow-up for an average 4.3 years with no significant clinical benefit for PCI, risks of death $(8.9 \%$ vs $9.1 \%$ ) and non-fatal MI (8.9\% vs $8.1 \%$ ) being nearly identical with medical therapy, while differences in unplanned revascularisation $(21.4 \%$ vs $30.7 \%$ ) and persistent angina (29\% vs $33 \%)$ were small and insignificant. The data support recent guideline recommendations for treatment of stable angina (see above), and have been used to challenge those clinicians who continue to offer PCI to patients not receiving OMT [45]. However, FAME-II has now provided some support for an early interventional approach in a randomised comparison of OMT and PCI using drug-eluting stents guided by fractional flow reserve (FFR) [46]. The study was stopped 17 months earlier than planned because the composite endpoint (all-cause mortality, non-fatal MI, urgent revascularisation) occurred in $4.3 \%$ of the PCI group compared with $12.7 \%$ of the non-PCI (OMT) group. Relief of angina was also more effective in the PCI group. Already, PCI guided by FFR has become a recommended strategy in stable coronary artery disease but some feel this is premature [47]. Thus, the treatment difference in FAME-II was driven solely by a reduction in urgent revascularisation (49 in the OMT alone group; 7 in the FFR-PCI group ( $\mathrm{HR}=0.13,95 \%$ CI $0.06-0.30)$ ), while the 33 deaths and non-fatal MIs were distributed fairly evenly between the groups. Moreover, the majority of patients undergoing 'urgent' revascularisation lacked objective findings of high-risk ischaemia or threshold biomarker elevations, raising concerns of biased selection of patients for invasive management during follow-up.
Nevertheless, the argument in favour of interventional management as an initial strategy in stable angina has undoubtedly been strengthened by FAME-II, but final answers to the debate may have to await the findings of the ongoing International Study of Comparative Health Effectiveness with Medical and Invasive Approaches (ISCHEMIA Trial; ClinicalTrials.gov number, NCT 01471522), comparing effects of revascularisation (PCI or CABG) combined with OMT, with OMT alone on cardiovascular death, or MI in patients with stable $\mathrm{CAD}$, and objective evidence of myocardial ischaemia.

\section{Coronary artery bypass surgery}

Updated US guidelines [48] have endorsed the NICE recommendation of a multidisciplinary team approach to adjudicating revascularisation decisions in patients with complex coronary disease, encouraging application of SYNTAX and other scoring systems in arriving at an appropriate decision [49]. The potential for CABG compared with PCI to improve prognosis in patients with left main and multivessel CAD is supported by recent cohort studies [50,51], and now available are the 5-year follow-up data from SYNTAX in which major adverse cardiac and cerebrovascular events (MACCE) were $26.9 \%$ in the CABG group and $37.3 \%$ in the PCI group, driven largely by lower rates of non-fatal myocardial infarction and repeat revascularisation for $C A B G$, with no significant difference in all-cause mortality and stroke compared with PCI [52]. The benefits of CABG were particularly evident in patients with intermediate and high SYNTAX scores, there being no significant difference in outcomes between revascularisation strategies for patients with low SYNTAX scores. Any question about the preferred revascularisation strategy in patients with diabetes and multivessel coronary artery disease has now been answered by the FREEDOM TRIAL which randomised 1900 patients on OMT to either PCI with drug-eluting stents or CABG [53]. After a median follow-up of 3.8 years, the primary outcome, a composite of death from any cause, non-fatal myocardial infarction, or non-fatal stroke, occurred in $26.6 \%$ of the PCI group and $18.7 \%$ of the CABG group. The authors concluded that CABG is superior to PCI in patients with diabetes and multivessel disease. There is less certainty about the preferred revascularisation strategy in left main coronary disease, the SYNTAX investigators reporting similar outcomes for PCI and CABG, a finding consistent with other contemporary studies that identify stenting as a reasonable strategy in appropriately selected cases, even though the need for repeat 
revascularisation is almost invariably higher compared with CABG [54,55].

Surgical technique has come under considerable scrutiny recently. Concerns about the potential adverse effects of endoscopic versus open saphenous vein harvesting have been based largely on a non-randomised cohort study of 1817 patients in whom rates of vein graft failure at 1 year were $47 \%$ vs $38 \%$, and rates of death, myocardial infarction or revascularisation at 3 years were $20.2 \%$ vs $17.4 \%$ for endoscopic versus open saphenous vein harvesting [56]. This led NICE to recommend caution in use of the endoscopic technique [57], but such concerns have now been allayed by the results of two large cohort studies. In the US study of 235,394 Medicare CABG patients in the Society of Thoracic Surgeons (STS), national database mortality rates were similar regardless of harvesting technique, while rates of harvest site complications were lower for the endoscopic technique [58]. A UK study of 4702 CABG patients reported similar findings with no differences in in-hospital mortality $(0.9 \%$ vs $1.1 \%, p=0.71)$ or midterm mortality (HR 1.04; 95\% CI 0.65-1.66) for endoscopic versus open vein harvesting [59].

Also under scrutiny have been the relative benefits of off-pump and on-pump CABG. Each has its proponents $[60,61]$, but the results of randomised outcome trials have failed to show any clear advantage for off-pump CABG, the 3-year results of the Best Bypass Surgery Trial showing no significant difference in the primary composite outcome of MACCE compared with on-pump CABG, but a tendency towards higher mortality [62]. This may reflect, at least in part, differences in graft patency rates favouring on-pump procedures, the ROOBY trial reporting rates of $91.4 \%$ vs $85.8 \%$ for arterial grafts and $80.4 \%$ vs $72.7 \%$ for saphenous vein grafts in on-pump compared with off-pump patients [63]. Particularly disappointing has been the failure of offpump surgery to reduce cerebral injury, but a randomised comparison of minimal (MECC) versus conventional (CECC) extracorporeal circulation in 64 patients undergoing $\mathrm{CABG}$ has been more promising [64]. MECC was associated with improved cerebral oxygen delivery during surgery, and neurocognitive performance at 3 months was better when compared with CECC.

\section{Remote ischaemic preconditioning for treatment of stable coronary disease}

Its proponents see remote ischaemic preconditioning (RIPC) as a useful and inexpensive means of improving outcomes across a range of car- diovascular disorders. They must be frustrated, therefore, by the technique's failure to penetrate clinical practice, conflicting reports of its efficacy and mechanistic uncertainty combining to undermine clinical confidence in the utility of RIPC. Some recent randomised trials have been favourable, reporting protection against contrastinduced nephropathy during cardiac catheterization [65] and reduction in myocardial injury during heart valve surgery [66]. Perhaps the most favourable has been a randomised trial of prehospital RIPC in 333 patients with STEMI who underwent primary PCI [67]. The group with RIPC showed a significant improvement in myocardial salvage index compared with the group without (0.75 vs 0.55 ) although the trial was not powered for coronary events. Against this must be set a negative trial of RIPC in a group of patients undergoing CABG [68], but this is unlikely to be the last word, and already a meta-analysis of nine studies including 704 patients has concluded that RIPC significantly reduces troponin release during CABG [69]. Mechanistic studies of interest include one crossover study in patients with stable coronary artery disease in which RIPC reduced platelet activation during exercise testing without protecting against ischaemic ECG changes [70]. In another study of forearm blood flow using venous plethysmography in healthy volunteers, RIPC protected against impaired endotheliumdependent vasomotor function induced by ischaemia [71]. However, this protection was unaffected by infusion of a bradykinin $\mathrm{B} 2$ receptor antagonist, leading the authors to conclude that bradykinin is not a mediator of RIPC.

\section{Prognostic biomarkers in stable CAD}

Circulating biomarkers. Interest in circulating cardiovascular biomarkers has never been higher, and methodological papers have been developed to alert researchers to the standards necessary for proper evaluation of their prognostic utility [72, 73]. However, a systematic review of 83 CRP studies was critical of their general quality and concluded that multiple types of reporting bias, and publication bias, make the magnitude of any independent association between CRP and prognosis among patients with stable coronary disease sufficiently uncertain that no clinical practice recommendations can be made [74]. The same authors were equally critical of $19 \mathrm{BNP}$ studies in patients with stable coronary disease, reporting that clinically useful measures of prediction and discrimination were generally unavailable, and concluding that the unbiased strength of association of BNP with prognosis in stable coronary disease 
is unclear [75]. The availability of high-sensitivity assays has seen renewed interest in troponins as markers of risk in stable coronary disease, a US study of 984 patients in the Heart and Soul Study reporting that each doubling in hs-cTnT level is associated with a $37 \%$ higher rate of cardiovascular events [76]. Meanwhile the PEACE investigators have reported that among 3623 patients with stable coronary artery disease, hs-cTNI is independently associated with cardiovascular death or heart failure (HR $1.88(1.33-2.66 ; p<0.001)$ ), the association with non-fatal myocardial infarction being weaker $(1.03-2.01 ; p=0.031)$ [77]. Evidence from CTCA suggests that clinically silent rupture of non-calcified plaque with subsequent microembolisation is a likely pathophysiological mechanism of troponin elevation [78] but it is too soon to know whether it will have a clinical role in the prognostic assessment of stable coronary artery disease. The same applies to the mid-regional portion of proadrenomedullin and other biomarkers currently under investigation [79].

\section{Vascular biomarkers}

Carotid intimamedia thickness (cIMT) is well established as a predictor of cardiovascular events in the general population and, more weakly, in patients with stable coronary artery disease [80]. Its predictive value may be enhanced by additional consideration of the extent of carotid plaque allowing derivation of the 'total burden score' which was shown by Chinese investigators to improve the prediction of the 5-year risk of cardiovascular endpoints compared with cIMT alone [81]. Certainly, the value of cIMT alone for cardiovascular risk prediction in the general population is under question following a large meta-analysis of participant-level data in 45,828 individuals in which cIMT added almost nothing to the Framingham Risk Score [82]. Further questions have been raised by another meta-analysis of participant-level data which included 36,984 individuals followed-up for an average of 7 years [83]. The investigators showed no association between progression of cIMT and risk of cardiovascular events, questioning the validity of using changes in cIMT as a surrogate endpoint in trials of cardiovascular risk.

\section{Calcium and parathyroid hormone}

Studies suggesting that people who take calcium supplements may be increasing their risk of myocardial infarction $[84,85]$ have stimulated interest in serum calcium and its relation to cardiovascular events in patients with CHD. A recent study has confirmed that vitamin $\mathrm{D}$, parathyroid hormone and calcium show association with car- diovascular risk factors in US adolescents [86], and now we have data in 1017 patients with stable coronary artery disease followed-up for a median of 8.1 years, suggesting that high calcium levels, but not high phosphate levels, might be associated with all-cause and cardiovascular mortality (HR 2.39-4.66) [87]. The mechanism of this association is unclear, but the demonstration in the same cohort of a similar association between high parathyroid hormone and cardiovascular mortality may implicate calcium mobilisation from bone on the causal pathway [88].

\section{Contributors}

SI and AT contributed equally to the preparation and writing of this review article.

\section{Competing interests}

None.

\section{Provenance and peer review}

Commissioned; internally peer reviewed.

\section{References}

[1] Nabel EG, Braunwald E. A tale of coronary artery disease and myocardial infarction. N Engl J Med 2012;366:54-63.

[2] Briffa T, Nedkoff L, Peeters A, et al. Discordant age and sex-specific trends in the incidence of a first coronary heart disease event in Western Australia from 1996 to 2007. Heart 2011;97:400-4.

[3] Vaartjes I, O'Flaherty M, Grobbee DE, et al. Coronary heart disease mortality trends in the Netherlands 1972-2007. Heart 2011;97:569-73.

[4] Schmidt M, Jacobsen JB, Lash TL, et al. 25 year trends in first time hospitalisation for acute myocardial infarction, subsequent short and long term mortality, and the prognostic impact of sex and comorbidity: a Danish nationwide cohort study. BMJ 2012;344:e356.

[5] Smolina K, Wright FL, Rayner M, et al. Determinants of the decline in mortality from acute myocardial infarction in England between 2002 and 2010: linked national database study. BMJ 2012;344:d8059.

[6] Nedkoff L, Briffa TG, Knuiman M, et al. Temporal trends in the incidence and recurrence of hospitalised atherothrombotic disease in an Australian population, 2000-07: data linkage study. Heart 2012;98:1449-56.

[7] Pearson-Stuttard J, Bajekal M, Scholes S, et al. Recent UK trends in the unequal burden of coronary heart disease. Heart 2012;98:1573-82.

[8] Kharazmi E, Dossus L, Rohrmann S, et al. Pregnancy loss and risk of cardiovascular disease: a prospective population-based cohort study (EPIC-Heidelberg). Heart 2011;97:49-54.

[9] Safford MM, Brown TM, Muntner PM, et al. Association of race and sex with risk of incident acute coronary heart disease events. JAMA 2012;308:1768-74.

[10] Williams ED, Stamatakis E, Chandola T, et al. Physical activity behaviour and coronary heart disease mortality among South Asian people in the UK: an observational longitudinal study. Heart 2011;97:655-9.

[11] Zaman MJ, Bhopal RS. New answers to three questions on the epidemic of coronary mortality in south Asians: 
incidence or case fatality? Biology or environment? Will the next generation be affected? Heart 2013;99:154-8.

[12] Fihn SD, Gardin JM, Abrams J, et al.; American College of Cardiology Foundation; American Heart Association Task Force on Practice Guidelines; American College of Physicians; American Association for Thoracic Surgery; Preventive Cardiovascular Nurses Association; Society for Cardiovascular Angiography and Interventions; Society of Thoracic Surgeons. ACCF/AHA/ACP/AATS/PCNA/ SCAI/STS Guideline for the diagnosis and management of patients with stable ischemic heart disease: a report of the American College of Cardiology Foundation/American Heart Association Task Force on Practice Guidelines, and the American College of Physicians, American Association for Thoracic Surgery, Preventive Cardiovascular Nurses Association, Society for Cardiovascular Angiography and Interventions, and Society of Thoracic Surgeons. J Am Coll Cardiol 2012;60:e44-164.

[13] Cooper A, Timmis A, Skinner J. Guideline Development Group. Assessment of recent onset chest pain or discomfort of suspected cardiac origin: summary of NICE guidance. BMJ 2010;340:c1118.

[14] Henderson RA, O'Flynn N; Guideline Development Group. Management of stable angina: summary of NICE guidance. Heart 2012;98:500-7.

[15] Taggart DP, Boyle R, de Belder MA, et al. The 2010 ESC/EACTS guidelines on myocardial revascularization. Heart 2011;97:445-6.

[16] Serruys PW, Morice MC, Kappetein AP, et al. Percutaneous coronary intervention versus coronary-artery bypass grafting for severe coronary artery disease. $N$ Engl $J$ Med 2009;360:961-72.

[17] D'Ascenzo F, Presutti DG, Picardi E, et al. Prevalence and non-invasive predictors of left main or three-vessel coronary disease: evidence from a collaborative international meta-analysis including 22740 patients. Heart 2012;98:914-9.

[18] Cacoub PP, Zeymer U, Limbourg T, et al. Effects of adherence to guidelines for the control of major cardiovascular risk factors on outcomes in the REduction of Atherothrombosis for Continued Health (REACH) Registry Europe. Heart 2011;97:660-7.

[19] Kotseva K, Jennings CS, Turner EL, et al. A survey of lifestyle, risk factor management and cardioprotective medication in patients with coronary heart disease and people at high risk of developing cardiovascular disease in the UK. Heart 2012;98:865-71.

[20] Stewart K, Walters M, Dawson J. Clopidogrel and modified-release dipyridamole for the prevention of occlusive vascular events (NICE technology appraisal guidance 90). Heart 2011;97:585-6.

[21] Mega JL, Close SL, Wiviott SD, et al. Cytochrome P-450 polymorphisms and response to clopidogrel. $\mathrm{N}$ Engl J Med 2009;360:354e62.

[22] Bouman HJ, Harmsze AM, van Werkum JW, et al. Variability in on-treatment platelet reactivity explained by CYP2C $19 * 2$ genotype is modest in clopidogrel pretreated patients undergoing coronary stenting. Heart 2011;97:1239-44.

[23] Hulot JS, Collet JP, Silvain J, et al. Cardiovascular risk in clopidogrel-treated patients according to cytochrome P450 2C19*2 loss-of-function allele or proton pump inhibitor coadministration: a systematic meta-analysis. J Am Coll Cardiol 2010;56:134e43.

[24] Zabalza M, Subirana I, Sala J, et al. Meta-analyses of the association between cytochrome CYP2C19 loss- and gain-of-function polymorphisms and cardiovascular outcomes in patients with coronary artery disease treated with clopidogrel. Heart 2012;98:100-8.
[25] Holmes MV, Perel P, Shah T, et al. CYP2C19 genotype, clopidogrel metabolism, platelet function, and cardiovascular events: a systematic review and meta-analysis. JAMA 2011;306:2704-14.

[26] Focks JJ, Brouwer MA, van Oijen MG, et al. Concomitant use of clopidogrel and proton pump inhibitors: impact on platelet function and clinical outcome - a systematic review. Heart 2013;99:520-7.

[27] Park KW, Kang J, Park JJ, et al. Amlodipine, clopidogrel and CYP3A5 genetic variability: effects on platelet reactivity and clinical outcomes after percutaneous coronary intervention. Heart 2012;98:1366-72.

[28] Li AY, Ng FH, Chan FK, et al. Effect of amlodipine on platelet inhibition by clopidogrel in patients with ischaemic heart disease: a randomised, controlled trial. Heart 2013;99:468-73.

[29] Nicholls SJ, Ballantyne CM, Barter PJ, et al. Effect of two intensive statin regimens on progression of coronary disease. N Engl J Med 2011;365:2078-87.

[30] The AIM-HIGH Investigators. Niacin in Patients with Low HDL Cholesterol Levels Receiving Intensive Statin Therapy. N Engl J Med 2011;365:2255-67.

[31] Barter PJ, Caulfield M, Eriksson M, et al. Effects of torcetrapib in patients at high risk for coronary events. $N$ Engl J Med 2007;357:2109-22.

[32] Schwartz GG, Olsson AG, Abt M, et al. Effects of dalcetrapib in patients with a recent acute coronary syndrome. N Engl J Med 2012;367:2089-99.

[33] Cannon CP, Shah S, Dansky HM, et al. Determining the Efficacy and Tolerability Investigators. Safety of anacetrapib in patients with or at high risk for coronary heart disease. N Engl J Med 2010;363:2406-15.

[34] Nicholls SJ, Brewer HB, Kastelein JJ, et al. Effects of the CETP inhibitor evacetrapib administered as monotherapy or in combination with statins on HDL and LDL cholesterol: a randomized controlled trial. JAMA 2011;306:2099-109.

[35] Neefjes LA, Ten Kate GJ, Rossi A, et al. CT coronary plaque burden in asymptomatic patients with familial hypercholesterolaemia. Heart 2011;97:1151-7.

[36] Cuchel M, Meagher EA, du Toit, et al. Efficacy and safety of a microsomal triglyceride transfer protein inhibitor in patients with homozygous familial hypercholesterolaemia: a single-arm, open-label, phase 3 study. Lancet 2013;381:40-6.

[37] Stein EA, Gipe D, Bergeron J, et al. Effect of a monoclonal antibody to PCSK9, REGN727/SAR236553, to reduce low-density lipoprotein cholesterol in patients with heterozygous familial hypercholesterolaemia on stable statin dose with or without ezetimibe therapy: a phase 2 randomised controlled trial. Lancet 2012;380:29-36.

[38] Raal F, Scott R, Somaratne R, et al. Low-density lipoprotein cholesterol-lowering effects of AMG 145, a monoclonal antibody to proprotein convertase subtilisin/kexin type 9 serine protease in patients with heterozygous familial hypercholesterolemia: the Reduction of LDL-C with PCSK9 Inhibition in Heterozygous Familial Hypercholesterolemia Disorder (RUTHERFORD) randomized trial. Circulation 2012;126:2408-17.

[39] Nherera L, Marks D, Minhas R, et al. Probabilistic cost-effectiveness analysis of cascade screening for familial hypercholesterolaemia using alternative diagnostic and identification strategies. Heart 2011;97:1175-81.

[40] Talmud PJ, Shah S, Whittall R, et al. Use of low-density lipoprotein cholesterol gene score to distinguish patients with polygenic and monogenic familial hypercholesterolaemia: a case-control study. Lancet 2013;381:1293-301.

[41] Gray J, Jaiyeola A, Whiting M, et al. Identifying patients with familial hypercholesterolaemia in primary care: an 
informatics-based approach in one primary care centre. Heart 2008;94:754-8.

[42] Boden WE, O'Rourke RA, Teo KK, et al. Optimal medical therapy with or without PCI for stable coronary disease. N Engl J Med 2007;356:1503-16.

[43] Weintraub WS, Spertus JA, Kolm P, et al. Effect of PCI on quality of life in patients with stable coronary disease. N Engl J Med 2008;359:677-87.

[44] Stergiopoulos K, Brown DL. Initial coronary stent implantation with medical therapy vs medical therapy alone for stable coronary artery disease: meta-analysis of randomized controlled trials. Arch Intern Med 2012;172:312-19.

[45] Borden WB, Redberg RF, Mushlin AI, et al. Patterns and intensity of medical therapy in patients undergoing percutaneous coronary intervention. JAMA 2011;305:1882-9.

[46] De Bruyne B, Pijls NH, Kalesan B, et al. FAME 2 Trial Investigators. Fractional flow reserve-guided PCI versus medical therapy in stable coronary disease. $N$ Engl J Med 2012;367:991-1001.

[47] Boden WE. COURAGE 5 years on: the message grows stronger. Heart 2012;98:1757-60.

[48] Hillis LD, Smith PK, Anderson JL, et al. American College of Cardiology Foundation; American Heart Association Task Force on Practice Guidelines; American Association for Thoracic Surgery; Society of Cardiovascular Anesthesiologists; Society of Thoracic Surgeons. ACCF/AHA Guideline for Coronary Artery Bypass Graft Surgery. A report of the American College of Cardiology Foundation/American Heart Association Task Force on Practice Guidelines. Developed in collaboration with the American Association for Thoracic Surgery, Society of Cardiovascular Anesthesiologists, and Society of Thoracic Surgeons. J Am Coll Cardiol 2011;58:e123-210.

[49] Farooq V, Brugaletta S, Serruys PW. Contemporary and evolving risk scoring algorithms for percutaneous coronary intervention. Heart 2011;97:1902-13.

[50] Hlatky MA, Boothroyd DB, Baker L, et al. Comparative effectiveness of multivessel coronary bypass surgery and multivessel percutaneous coronary intervention: a cohort study. Ann Intern Med 2013;158:727-34.

[51] Weintraub WS, Grau-Sepulveda MV, Weiss JM, et al. Comparative effectiveness of revascularization strategies. N Engl J Med 2012;366:1467-76.

[52] Mohr FW, Morice MC, Kappetein AP, et al. Coronary artery bypass graft surgery versus percutaneous coronary intervention in patients with three-vessel disease and left main coronary disease: 5 -year follow-up of the randomised, clinical SYNTAX trial. Lancet 2013;381:629-38.

[53] Farkouh ME, Domanski M, Sleeper LA, et al. FREEDOM Trial Investigators. Strategies for multivessel revascularization in patients with diabetes. $N$ Engl $J$ Med 2012;367:2375-84.

[54] Chieffo A, Meliga E, Latib A, et al. Drug-eluting stent for left main coronary artery disease. The DELTA registry: a multicenter registry evaluating percutaneous coronary intervention versus coronary artery bypass grafting for left main treatment. JACC Cardiovasc Interv 2012;5:718-27.

[55] Chang K, Koh YS, Jeong SH, et al. Long-term outcomes of percutaneous coronary intervention versus coronary artery bypass grafting for unprotected left main coronary bifurcation disease in the drug-eluting stent era. Heart 2012; 98:799-805.

[56] Lopes RD, Hafley GE, Allen KB, et al. Endoscopic versus open vein-graft harvesting in coronary-artery bypass surgery. N Engl J Med 2009;361:235-44.
[57] Barnard JB, Keenan DJ. National Institute for Health and Clinical Excellence. Endoscopic saphenous vein harvesting for coronary artery bypass grafts: NICE guidance. Heart 2011;97:327-9.

[58] Williams JB, Peterson ED, Brennan JM, et al. Association between endoscopic vs open vein-graft harvesting and mortality, wound complications, and cardiovascular events in patients undergoing CABG surgery. JAMA 2012;308:475-84.

[59] Grant SW, Grayson AD, Zacharias J, et al. What is the impact of endoscopic vein harvesting on clinical outcomes following coronary artery bypass graft surgery? Heart 2012;98:60-4.

[60] Pepper JR. NICE guidance for off-pump CABG: keep the pump primed. Heart 2011;97:1728-30.

[61] Falk V, Taggart DP. NICE guidance for off-pump CABG: turn off the pump. Heart 2011;97:1731-3.

[62] Møller CH, Perko MJ, Lund JT, et al. Three-year follow-up in a subset of high-risk patients randomly assigned to off-pump versus on-pump coronary artery bypass surgery: the Best Bypass Surgery trial. Heart 2011;97:907-13.

[63] Hattler B, Messenger JC, Shroyer AL, et al. Veterans Affairs Randomized On/Off Bypass (ROOBY) Study Group. Off-Pump coronary artery bypass surgery is associated with worse arterial and saphenous vein graft patency and less effective revascularization: results from the Veterans Affairs Randomized On/Off Bypass (ROOBY) trial. Circulation 2012;125:2827-35.

[64] Anastasiadis K, Argiriadou H, Kosmidis MH, et al. Neurocognitive outcome after coronary artery bypass surgery using minimal versus conventional extracorporeal circulation: a randomised controlled pilot study. Heart 2011;97:1082-8.

[65] Er F, Nia AM, Dopp H, et al. Ischemic preconditioning for prevention of contrast medium-induced nephropathy: randomized pilot RenPro Trial (Renal Protection Trial). Circulation 2012;126:296-303.

[66] Xie JJ, Liao XL, Chen WG, et al. Remote ischaemic preconditioning reduces myocardial injury in patients undergoing heart valve surgery: randomised controlled trial. Heart 2012;98:384-8.

[67] Bøtker HE, Kharbanda R, Schmidt MR, et al. Remote ischaemic conditioning before hospital admission, as a complement to angioplasty, and effect on myocardial salvage in patients with acute myocardial infarction: a randomised trial. Lancet 2010;375:727-34.

[68] Rahman IA, Mascaro JG, Steeds RP, et al. Remote ischemic preconditioning in human coronary artery bypass surgery: from promise to disappointment? Circulation 2010;122(11 Suppl):S53-9.

[69] D'Ascenzo F, Cavallero E, Moretti C, et al. Remote ischaemic preconditioning in coronary artery bypass surgery: a meta-analysis. Heart 2012;98:1267-71.

[70] Battipaglia I, Scalone G, Milo M, et al. Upper arm intermittent ischaemia reduces exercise-related increase of platelet reactivity in patients with obstructive coronary artery disease. Heart 2011;97:1298-303.

[71] Pedersen CM, Schmidt MR, Barnes G, et al. Bradykinin does not mediate remote ischaemic preconditioning or ischaemia-reperfusion injury in vivo in man. Heart 2011;97:1857-61.

[72] Moons KG, Kengne AP, Woodward M, et al. Risk prediction models: I. Development, internal validation, and assessing the incremental value of a new (bio)marker. Heart 2012;98:683-90.

[73] Moons KG, Kengne AP, Grobbee DE, et al. Risk prediction models: II. External validation, model updating, and impact assessment. Heart 2012;98:691-8. 
[74] Hemingway H, Philipson P, Chen R, et al. Evaluating the quality of research into a single prognostic biomarker: a systematic review and meta-analysis of 83 studies of C-reactive protein in stable coronary artery disease. PLoS Med 2010;7: e1000286.

[75] Sutaria S, Philipson P, Fitzpatrick NK, et al. Translational phases of evidence in a prognostic biomarker: a systematic review and meta-analysis of natriuretic peptides and the prognosis of stable coronary disease. Heart 2012;98:615-22.

[76] Beatty AL, Ku IA, Christenson RH, et al. High-sensitivity cardiac troponin $\mathrm{T}$ levels and secondary events in outpatients with coronary heart disease from the heart and soul study. JAMA Intern Med 2013;173:763-9.

[77] Omland T, Pfeffer MA, Solomon SD, et al. Prognostic value of cardiac troponin I measured with a highly sensitive assay in patients with stable coronary artery disease. J Am Coll Cardiol 2013;61:1240-9.

[78] Korosoglou G, Lehrke S, Mueller D, et al. Determinants of troponin release in patients with stable coronary artery disease: insights from CT angiography characteristics of atherosclerotic plaque. Heart 2011;97:823-31.

[79] Brouwers FP, de Boer RA, van der Harst P, et al. Influence of age on the prognostic value of mid-regional pro-adrenomedullin in the general population. Heart 2012;98:1348-53.

[80] Held C, Hjemdahl P, Eriksson SV, et al. Prognostic implications of intima-media thickness and plaques in the carotid and femoral arteries in patients with stable angina pectoris. Eur Heart J 2001;22:62-72.

[81] Xie W, Liang L, Zhao L, et al. Combination of carotid intima-media thickness and plaque for better pre- dicting risk of ischaemic cardiovascular events. Heart 2011;97:1326-31.

[82] Den Ruijter HM, Peters SA, Anderson TJ, et al. Common carotid intima-media thickness measurements in cardiovascular risk prediction: a meta-analysis. JAMA 2012;308:796-803.

[83] Lorenz MW, Polak JF, Kavousi M, et al. Carotid intima-media thickness progression to predict cardiovascular events in the general population (the PROG-IMT collaborative project): a meta-analysis of individual participant data. Lancet 2012;379:2053-62.

[84] Bolland MJ, Avenell A, Baron JA, et al. Effect of calcium supplements on risk of myocardial infarction and cardiovascular events: meta-analysis. BMJ 2010;341:c3691.

[85] Li K, Kaaks R, Linseisen J, et al. Associations of dietary calcium intake and calcium supplementation with myocardial infarction and stroke risk and overall cardiovascular mortality in the Heidelberg cohort of the European Prospective Investigation into Cancer and Nutrition study (EPIC-Heidelberg). Heart 2012;98:920-5.

[86] Williams DM, Fraser A, Lawlor DA. Associations of vitamin $\mathrm{D}$, parathyroid hormone and calcium with cardiovascular risk factors in US adolescents. Heart 2011;97:315-20.

[87] Grandi NC, Brenner H, Hahmann H, et al. Calcium, phosphate and the risk of cardiovascular events and all-cause mortality in a population with stable coronary heart disease. Heart 2012;98:926-33.

[88] Grandi NC, Breitling LP, Hahmann H, et al. Serum parathyroid hormone and risk of adverse outcomes in patients with stable coronary heart disease. Heart 2011;97:1215-21. 